\title{
Output response identification in a multistable system for piezoelectric energy harvesting
}

\author{
Peter Harris $^{1}$, Mustafa Arafa ${ }^{2}$, Grzegorz Litak ${ }^{3,4, a}$, Chris R. Bowen ${ }^{1}$, and Joanna Iwaniec ${ }^{5}$ \\ 1 Department of Mechanical Engineering, University of Bath, Bath BA2 7AY, UK \\ 2 Mechanical Engineering Department, American University in Cairo, AUC Avenue, P.O. Box 74, 11835 New Cairo, Egypt \\ 3 Faculty of Mechanical Engineering, Lublin University of Technology, Nadbystrzycka 36, 20-618 Lublin, Poland \\ 4 Department of Process Control, AGH University of Science and Technology, Mickiewicz Alley 30, 30-059 Krakow, Poland \\ 5 Department of Robotics and Mechatronics, AGH University of Science and Technology, Mickiewicz Alley 30, 30-059 Krakow, \\ Poland
}

Received 17 October 2016 / Received in final form 6 December 2016

Published online 25 January 2017

(C) The Author(s) 2017. This article is published with open access at Springerlink.com

\begin{abstract}
In this paper we examine in detail the multiple responses of a novel vibrational energy harvester composed of a vertical bistable beam whose complex non-linear behavior is tuned via magnetic interaction. The beam was excited horizontally by a harmonic inertial force while mechanical vibrational energy is converted to electrical power through a piezoelectric element. The bistable laminate beam coupled to the piezoelectric transducer showed a variety of complex responses in terms of the beam displacement and harvested power output. The range of vibration patterns in this non-linear system included single-well oscillations and snap-through vibrations of periodic and chaotic character. Harvested power was found to be strongly dependent on the vibration pattern with nonlinearities providing a broadband response for energy harvesting. Wavelet analysis of measured voltage, displacement and velocity time histories indicated the presence of a variety of nonlinear periodic and also chaotic phenomena. To measure the complexity of response time series we applied phase portraits and determine stroboscopic points and multiscale entropy. It is demonstrated that by changing parameters such as the magnetic interaction, the characteristics of the bistable laminate harvester, such as the natural frequency, bandwidth, vibration response and peak power can be readily tailored for harvesting applications.
\end{abstract}

\section{Introduction}

Piezoelectric vibration energy harvesting from ambient sources is a promising technology for distributed power generation of small autonomic devices such as wireless sensor networks and recharging batteries [1-4]. Much research effort has focused upon the refinement of devices operating in the linear mode and some commercial devices are available. These devices require a close matching between the natural frequency of the device and the input source. Consequently, these solutions are only really effective in context where the frequency of the input source is mostly constant.

Various nonlinear effects have been employed to mechanical resonators in order to broaden the frequency range of energy harvesters [5-7]. As a result, research into the use of multistable structures is expanding $[8,9]$. These structures exhibit two or more equilibrium states and the nonlinear response to vibrational excitation can be tailored to provide large-amplitude, cross-well oscilla-

\footnotetext{
a e-mail: g.litak@pollub.pl
}

tions which allow for a broader band response than that of linear devices [10].

Multistability can be induced through several means such as magnetic interactions [11], post-buckled structural members [12], pre-stressed metal diaphragms or polymeric laminae $[13,14]$, and thermal coefficient disparity [15]. This work examines bistable laminates for broadband energy harvesting where both laminate properties and a tip magnet are used to tune the harvester response. Initial work was carried out by Betts and Arrieta [16-18] in which square plate bistable laminates were built from asymmetrically laid carbon fiber pre-preg stacks with piezoelectric patches adhered for transduction of strain energy into electrical energy. Cantilever-type harvesters were also considered because of their more straightforward strain fields, lower natural frequencies and decreased acceleration levels needed for initiation of cross-well oscillations [19-22]. In this work, a novel bistable laminate harvester configuration is considered where the device consists of a bistable cantilever beam with repelling magnets of adjustable distance so that the contribution of the magnetic field to the overall strain energy landscape 
of the device can be controlled. By changing this gap, the characteristics of the harvester, such as the natural frequency, bandwidth, and peak power can be affected. Previous effort [23-25] has examined the highly energetic cross-well dynamics of bistable plates and cantilever systems, and in this paper the doubly bistable system is experimentally characterised and the response is examined in detail by a variety of signal processing methods. The experimental results such as mechanical resonator velocity, displacement as well as voltage output of the system are studied using wavelets and multiscale entropy in order to better understand the dynamics of this complicated system. This approach provides a new route to tailor the non-linear response of the energy harvester in response to the mechanical vibrations that are being harvested.

\section{Experimental characterisation}

Since the benefit of a bistable energy harvesting system stems from its nonlinearity and broadband response, the major contributing factors giving rise to device nonlinearities are initially investigated. The main factors that contribute to such a response are (i) the forces resulting simply from the magnetic interactions and (ii) the bistable strain energy landscape of the system. Both the magnetic interaction and nonlinear strain energy landscapes are characterized by measurement of their force-deflection functions experimentally. The construction of the bistable energy harvester is then detailed. Since the extent of nonlinearity due to the presence of the magnet is tunable by adjusting the separation distance between the magnets, the effects of this upon the energy harvesting capability is then investigated by undertaking sweeps through the frequency range of interest with vibrations at constant acceleration. The control of the measurement system is presented as well as the measurement of the root mean squared (rms) power for each frequency/acceleration combination in the explored parametric space. These sweeps are evaluated against a control case where there is no magnetic interaction.

\subsection{Manufacture of the bistable cantilever energy harvester}

The bistable cantilever energy harvester was manufactured from carbon fibre reinforced plastic (CFRP) plies of unidirectional UDM 194 carbon fiber in an M21 epoxy matrix. A smart materials piezoelectric M8528-P2 Macro Fiber Composite (MFC) patch was attached to the bistable cantilever using an epoxy adhesive for transduction of mechanical strain energy into electrical energy, as shown in Figure 1. The MFC is made up of a lead zirconate titanate (PZT) ferroelectric ceramic which is polarised through the thickness. This is in contrast to most MFC configurations with an interdigital electrode (IDE) where the polarization direction is along the fibre length and is characterized by a low efficiency. In this case the M8528-P2 device is polarized through thickness by continuous upper and lower electrodes composed of fine copper mesh. Compared to an IDE based device such a configuration has (i) an uniform electric field distribution through the ferroelectric material (ii) a high device capacitance $(C)$, leading to low peak voltages $(V)$ as a result of the piezoelectric charge $(Q=C V)$ and (iii) a low electrical impedance $(i \omega C)$ due to the high device capacitance [24].

As a result of the construction of the MFC patch with fibers running along the longitudinal axis and being embedded in a polymer matrix, the ensuing anisotropy requires that the directionality of the patch to be also stated in any stacking sequence. The cantilever used had a free length of $210 \mathrm{~mm}$ with an additional $30 \mathrm{~mm}$ for clamping between two aluminum plates. The width of the laminate was $60 \mathrm{~mm}$, and the ply thickness was between $0.185 \mathrm{~mm}$ and $0.195 \mathrm{~mm}$. In the clamped region (left hand side of Fig. 1a), two additional plies were included to ensure the beam is locally monostable so that clamping would not restrict the curvature of the cantilever. Thus, the stacking sequence at the clamped end is $\left[0 / 0 / 90 / 90 / 0 / 0 / 0_{M F C}\right]_{T}$ but the sequence for the majority of the beam is $\left[90 / 90 / 0 / 0 / 0_{M F C}\right]$. A small fixture was adhered at the end of the cantilever to receive the magnet, as shown on the right of Figure 1a. To investigate the change of the energy harvesting characteristic with respect to magnetic separation distance, a rigid metal frame was constructed to hold a movable magnet of the same specification as that in the cantilever tip. This magnet was mounted at the end of a threaded bolt for simple and precise vertical positioning, as shown in Figures $1 \mathrm{~b}$ and $1 \mathrm{c}$.

\subsection{Measurement of transverse magnetic force as a function of magnet separation}

The transverse magnetic force as a function of the separation distance between the magnets was measured by mounting a neodymium magnet vertically and fixing it to a digital load cell (see Fig. 2a). The second magnet was fixed to a vertical rail such that the gap between the magnets in the horizontal direction and their relative displacement in the vertical direction could be adjusted, as shown in Figure 2a. A range of magnet separations (gap widths) at different vertical displacements were recorded, and the measured forces are shown in Figure $2 \mathrm{~b}$. These forces were used to tune the response of a bistable laminate.

\subsection{Measurement of the force-deflection characteristics of the bistable cantilever and its hysteresis}

The force deflection curve of the bistable cantilever was measured on an Instron 3365 tensile tester with a $100 \mathrm{~N}$ load cell. In Figures 3a and 3b, a positive displacement is defined to be displacement towards the region II (in Fig. $3 \mathrm{~b}$ ), and curved state (see Figs. 1c and 3a) of the region III and IV (Fig. 3b). A negative displacement is in the opposite direction (region I in Fig. 3b).

The cantilever was immobilized and the cross head was moved downwards at a rate of approximately $4 \mathrm{~mm} / \mathrm{s}$ and then returned upwards to the starting position, 


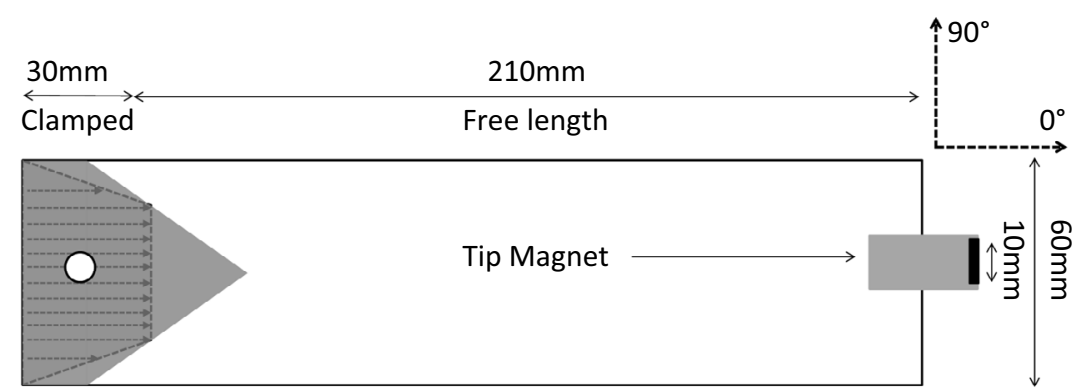

(a)

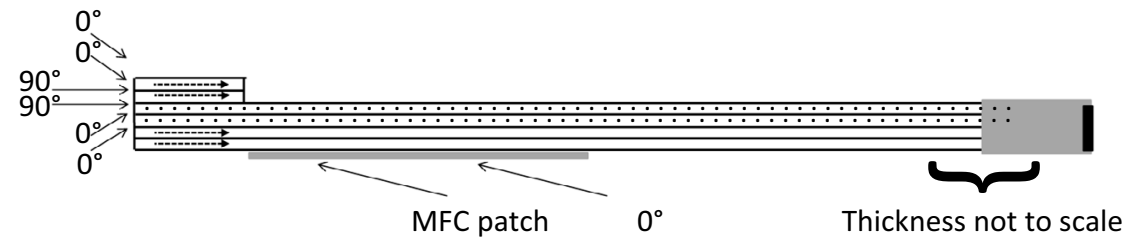

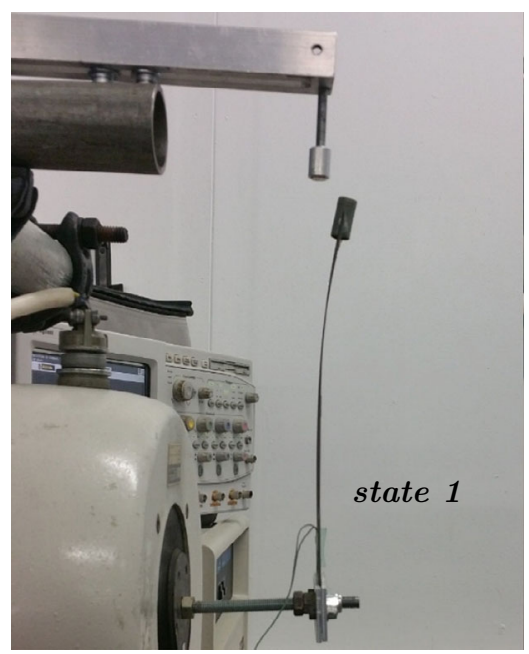

(b)

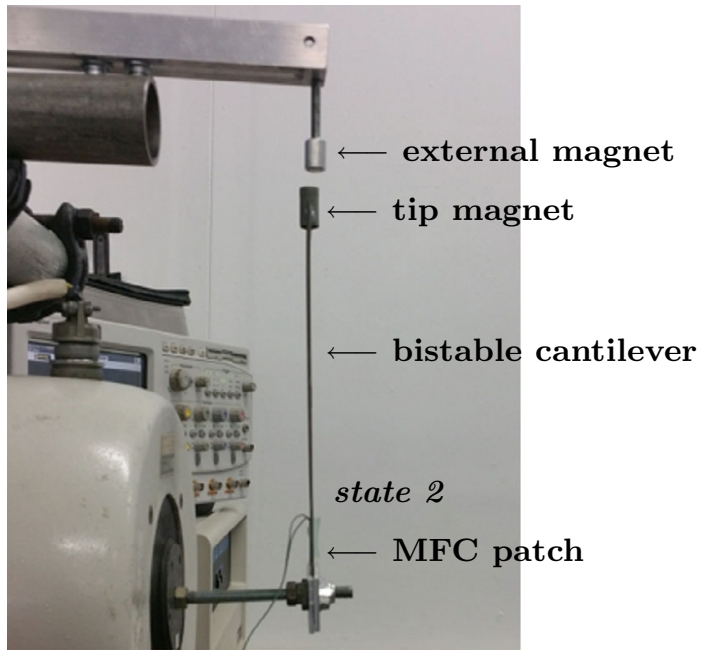

(c)

Fig. 1. (a) Detail of the ply orientations and magnet location on piezoelectric macro fiber composite (MFC), (b) stable state 1, and $(\mathrm{c})$ stable state 2 .

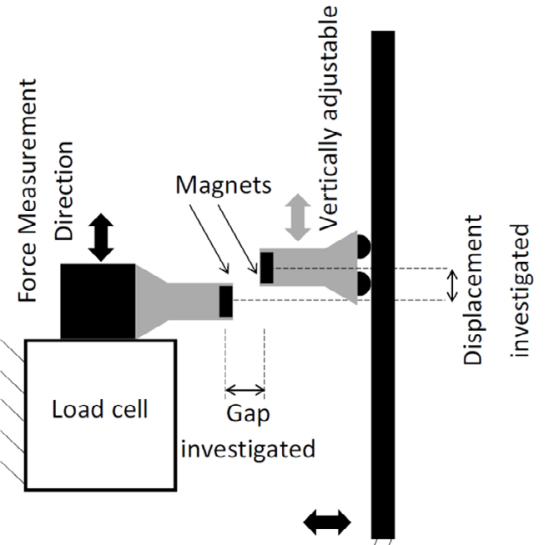

(a) Horizontally adjustable

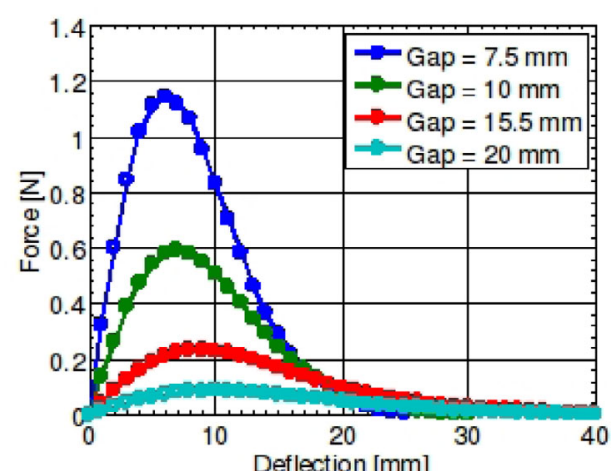

(b)

Fig. 2. (a) Schematic of method used for measuring magnetic force-deflection function and (b) the force deflection function of the magnets at the indicated gap distances and displacements. 

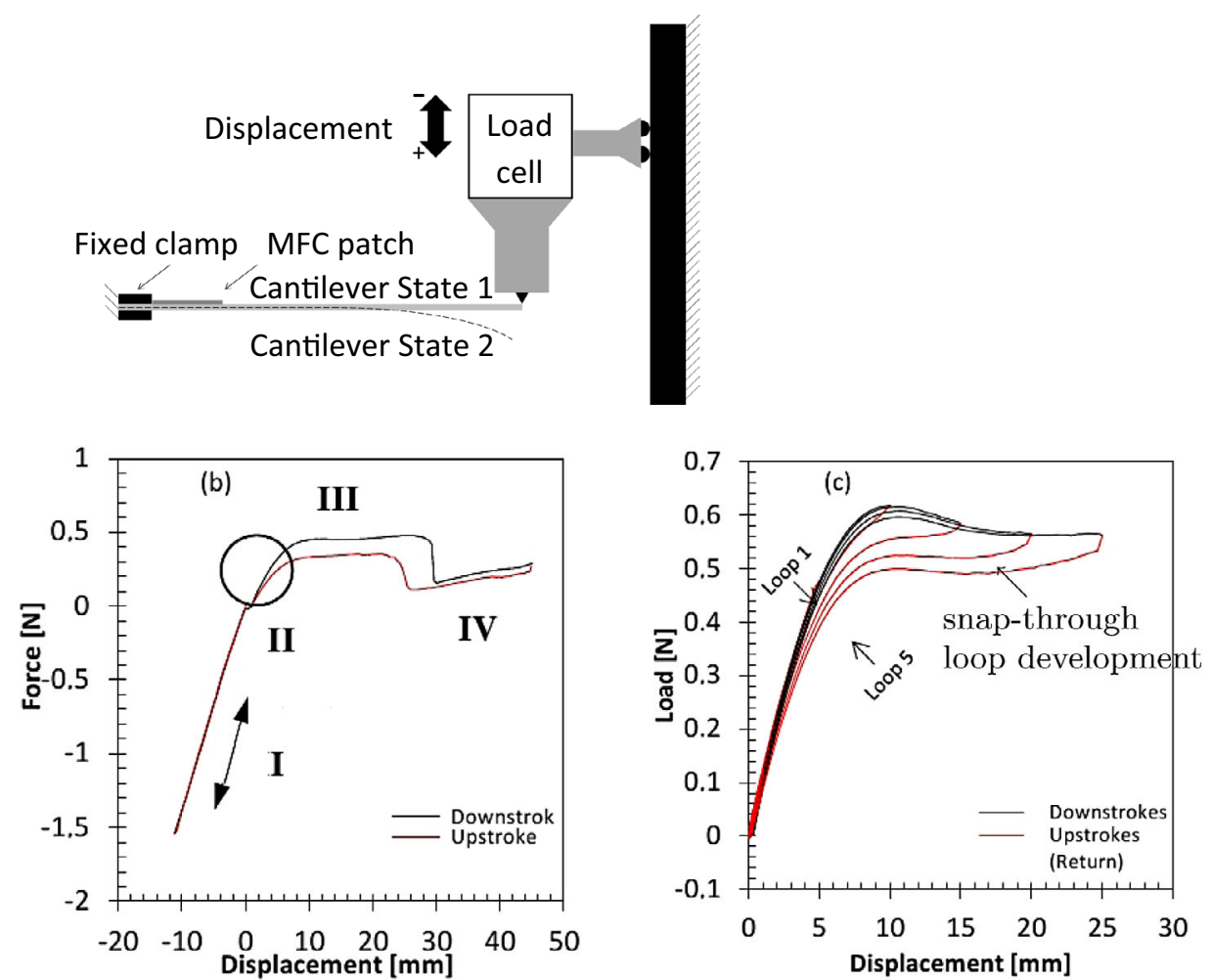

Fig. 3. (a) Schematic of bistable cantilever's orientation used during tensile testing. (b) Composited displacement-force function for the bistable cantilever harvester. Region I is the region in which the cantilever is bent in the direction opposite to snap through (negative displacements). Region II is the region magnified in (c). Region III is the snap-through and snap-back section. Region IV is the cantilever being stressed in state 2. (c) Loading/unloading curves just prior to complete snap-through.

recording the load cell force on both the downward and upward motions. For the positive displacement portion of the graph, 20 up-and-down loops were compiled to produce Figure $3 \mathrm{~b}$, and nine loops were undertaken for the negative side. To compile multiple results for robustness, each individual loop was first considered separately. As a result of slight differences at each time step, the loops could not be simply averaged together. Thus, for each small quasistatic loop, a linear interpolation was done between all the measured points such that the interpolated points all had exactly the same $x$-coordinate, but with a $y$-coordinate specific to that particular loop. These interpolated loops were then averaged together to produce Figure 3b. To investigate the hysteresis up to the point of complete snapthrough (Fig. 3c), first a cross-head displacement of $5 \mathrm{~mm}$ was undertaken and the displacement was then gradually increased by $5 \mathrm{~mm}$ increments to $25 \mathrm{~mm}$ (loops 1-5 in Fig. 3c). A snap-through phenomenon is related to the transition between the two equilibria (states 1 and 2 in Figs. $2 \mathrm{~b}$ and $2 \mathrm{c}$ ) of the bistable laminate beam. This leads to a larger displacement or vibration amplitude.

\subsection{RMS power and frequency sweeps}

For the energy harvesting system, four magnet separation distances of 20,15.5, 10 and $7.5 \mathrm{~mm}$ were investigated and compared against the condition of no magnetic interaction, as shown in Figure 4. For the case of no magnetic

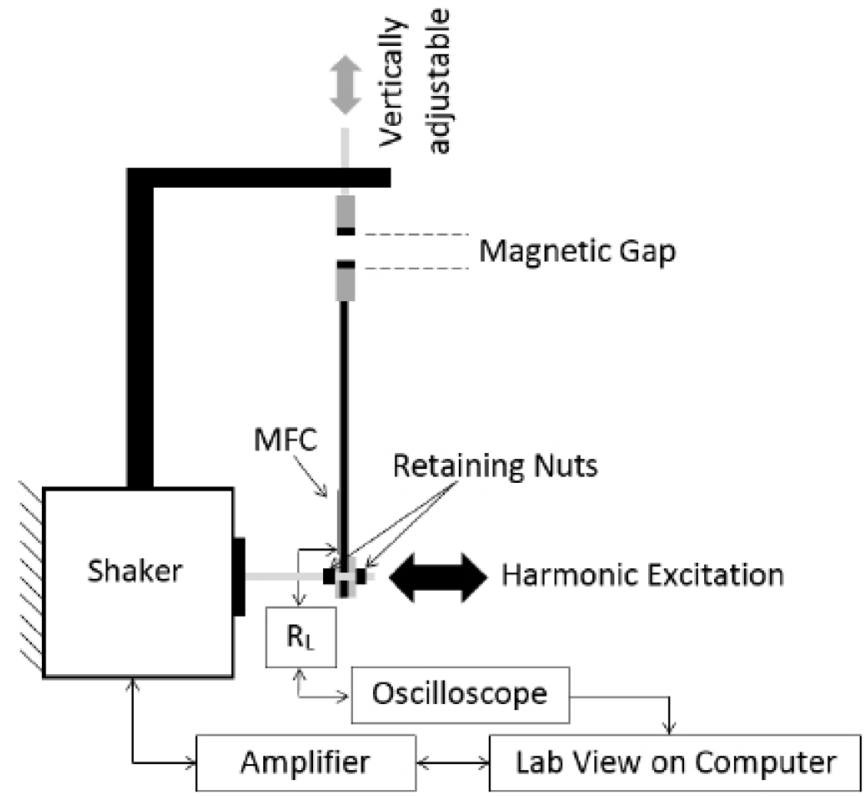

Fig. 4. Supporting frame with vertically adjustable magnet and electromechanical shaker. Control and voltage acquisition equipment schematically represented.

interaction, the magnet was present within the cantilever's tip to maintain experimental consistency, but the second fixed position magnet was removed. At each of the magnet 
separations, the harvester (see Fig. 4) was harmonically excited from 12 to $30 \mathrm{~Hz}$ in increments of $0.2 \mathrm{~Hz}$. At each frequency step $(\Delta \omega)$, a voltage signal over a load resistor was measured using an Agilent oscilloscope and an rms power was calculated. The shaker was calibrated using a Lab View routine to deliver constant acceleration at required frequencies. The LDS 455 shaker was calibrated by exciting the shaker with a sine wave of controlled amplitude and frequency running through the LDS PA 1000 amplifier. A matrix of voltage amplitudes and frequencies was sent to the shaker, and the response was measured using a Polytech laser differential Vibrometer which measured the velocity using the PSV-400-M4 scanning head, OFV-5000 controller and VD-09 velocity decoder. The peak acceleration was calculated from the velocity data and saved in the calibration file using a LabView routine running on a computer. When a specific acceleration is required at a desired frequency, another routine interpolates between the nearest voltages to deliver the needed acceleration magnitude. At each frequency, the acquisition system ignores the first $0.2 \mathrm{~s}$ of data to allow the harvester to be in a steady state. Approximately five seconds of voltage data is then acquired by the oscilloscope over the load resistor, and the rms voltage is determined and the rms power is calculated. Each of the forcing levels was repeated five times, and the results were averaged for robustness. The results of these power sweeps appear in Figure 5. The matching load resistor was found using impedance matching where $R_{L}=1 / 2 \pi f C$ [21]. Here, $R_{L}$ is the load resistance value required to maximize power output, $C$ is the capacitance of the MFC patch, and $f$ is the natural frequency of the cantilever. The patch capacitance was measured to be $156.5 \mathrm{nF}$, and the natural frequency was $21+/-1 \mathrm{~Hz}$, giving the value of the optimum load resistor to be $48 \mathrm{k} \Omega$, which was used throughout the harvesting experiments.

The frequency sweeps shown in Figure 5 have been carried out with increasing frequency. For the case of no magnet, i.e. an infinitely large magnetic gap, snap through of the cantilever was observed at $3 g$ (Fig. $5 \mathrm{c}$ ). As the magnetic separation was decreased, the acceleration required to induce snap-through oscillations was decreased. At a magnetic separation of 15.5 and $10 \mathrm{~mm}$, snap through was observed at $2 g$ (Fig. 5b). Interestingly, at a magnetic gap of $10 \mathrm{~mm}$, the potential fields of the magnets and thermal stresses coincided such that the system was effectively tristable. At the magnetic gap of $7.5 \mathrm{~mm}$, the magnetic interaction was strong enough that no snap through was observed at any forcing level. In addition to the results shown, the decreasing frequency sweeps were recorded but are not shown for sake of clarity and brevity.

The peak power outputs for the different forcing levels and different gap distances are shown in Table 1 for both up-sweep (increasing frequency) and down-sweep (decreasing frequency). Where the addition of magnetic interaction has increased the peak power level with respect to the baseline of no magnet, or led to a reduction in harvested power, this is shown by the + and - signs in the parentheses in Table 1. A trend emerges wherein the closer magnetic separations, in particular $10 \mathrm{~mm}$ and $15.5 \mathrm{~mm}$,
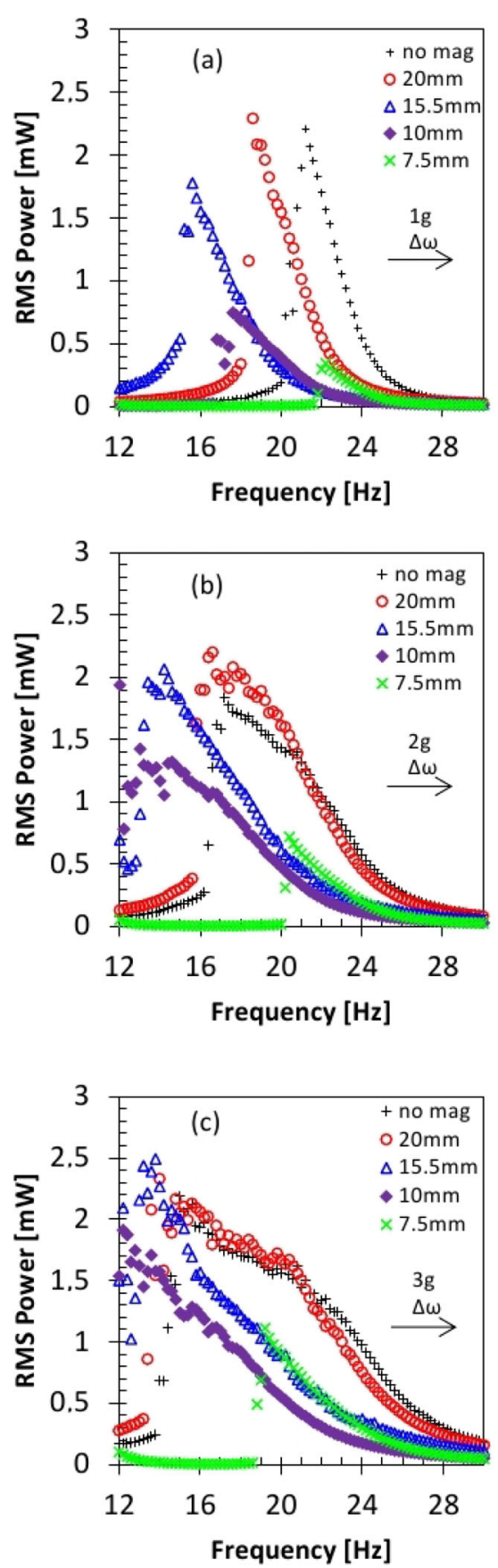

Fig. 5. rms power from frequency sweeps at various acceleration amplitude: (a) $1 g$, (b) $2 g$, and (c) $3 g$.

improve the peak power output at higher excitation levels of $2 g$ and $3 g$. The broadband energy harvesting capability of the system is measured based on the bandwidth at which at least half the maximal power output is generated. Since the lower frequency bound for half-maximum power could not always be observed the higher-frequency extent of the harvested power was considered. Given the asymmetry of the shape of the rms power generated, this is the more informative dimension as the onset of power generation is usually sudden and then gradually decreases with increasing frequency. 
Table 1. Effect of magnetic gap distance on peak power output for the considered $g$ levels for increasing (upper panel) and decreasing (lower panel) frequency sweeps.

\begin{tabular}{cccc}
\hline $\begin{array}{c}\text { Separation } \\
{[\mathrm{mm}]}\end{array}$ & $\begin{array}{c}\text { Max power at } 1 g \\
\text { and bandwidth }\end{array}$ & $\begin{array}{c}\text { Max power at } 2 g \\
\text { and bandwidth }\end{array}$ & $\begin{array}{c}\text { Max power at } 3 g \\
\text { and bandwidth }\end{array}$ \\
\hline No magnet & $2.21[\mathrm{~mW}] 1.6 \mathrm{~Hz}$ & $1.84[\mathrm{~mW}] 5.4 \mathrm{~Hz}$ & $2.19[\mathrm{~mW}] 8.2 \mathrm{~Hz}$ \\
20 & $(+3.73 \%) 2.0 \mathrm{~Hz}$ & $(+19.58 \%) 4.8 \mathrm{~Hz}$ & $(+6.59 \%) 8.0 \mathrm{~Hz}$ \\
15.5 & $(-19.54 \%) 2.2 \mathrm{~Hz}$ & $(+12.17 \%) 4.0 \mathrm{~Hz}$ & $(+14.08 \%) 4.0 \mathrm{~Hz}$ \\
10 & $(-66.27 \%) 2.4 \mathrm{~Hz}$ & $(+5.32 \%) 5.2 \mathrm{~Hz}$ & $(+19.07 \%) 5.2 \mathrm{~Hz}$ \\
7.5 & $(-84.32 \%) 1.2 \mathrm{~Hz}$ & $(-60.87 \%) 2.0 \mathrm{~Hz}$ & $(-49.03 \%) 2.6 \mathrm{~Hz}$ \\
\hline \multicolumn{5}{|}{} \\
\hline Separation & Max power at $1 g$ & Max power at $2 g$ & Max power at $3 g$ \\
{$[\mathrm{~mm}]$} & and bandwidth & and bandwidth & and bandwidth \\
\hline No magnet & $2.44[\mathrm{~mW}] 2.0 \mathrm{~Hz}$ & $1.95[\mathrm{~mW}] 6.6 \mathrm{~Hz}$ & $2.19[\mathrm{~mW}] 8.4 \mathrm{~Hz}$ \\
20 & $(-7.78 \%) 2.4 \mathrm{~Hz}$ & $(+13.87 \%) 5.2 \mathrm{~Hz}$ & $(+3.25 \%) 8.6 \mathrm{~Hz}$ \\
15.5 & $(-22.19 \%) 3.4 \mathrm{~Hz}$ & $(+8.78 \%) 5.4 \mathrm{~Hz}$ & $(+11.00 \%) 5.8 \mathrm{~Hz}$ \\
10 & $(-69.19 \%) 2.6 \mathrm{~Hz}$ & $(-7.29 \%) 5.0 \mathrm{~Hz}$ & $(+105.14 \%) 5.6 \mathrm{~Hz}$ \\
7.5 & $(-85.11 \%) 1.6 \mathrm{~Hz}$ & $(-63.38 \%) 2.6 \mathrm{~Hz}$ & $(-47.94 \%) 3.6 \mathrm{~Hz}$ \\
\hline
\end{tabular}

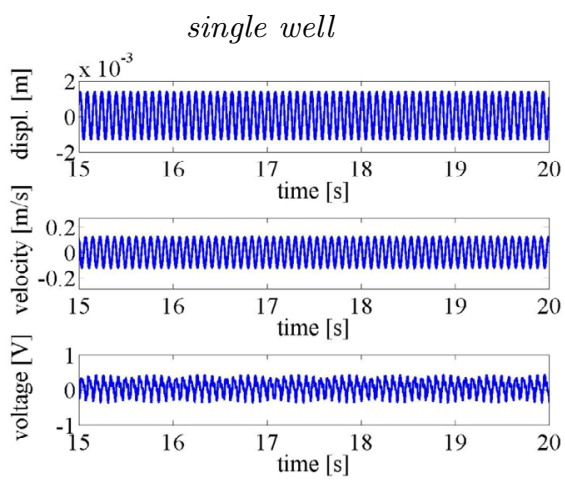

(a)

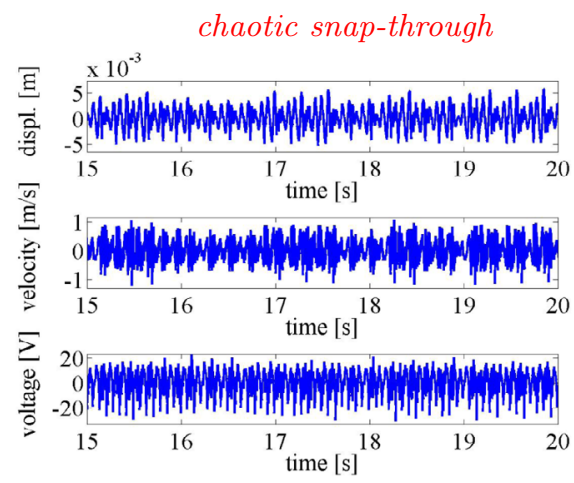

(b)
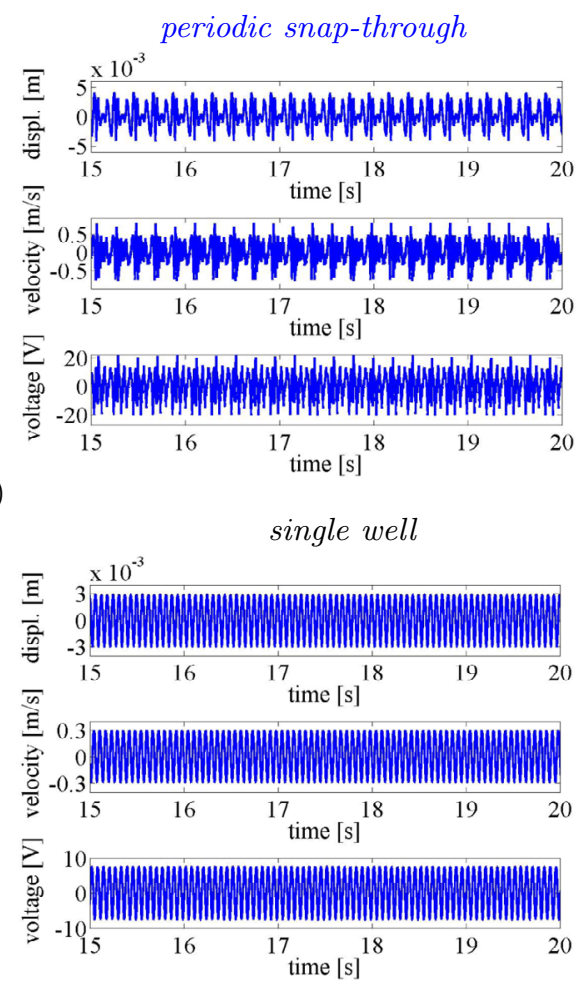

(d)

Fig. 6. Displacement, velocity and voltage time series for cases (a)-(d) described in Tables 2 and 3. The sampling frequency was $500 \mathrm{~Hz}$ with the laser beam oriented to the upper half of the cantilever.

\subsection{Selected time series of the system response}

It is clear from Figure 6 that our multistable beam is characterized by complex and nonlinear responses. Such systems exhibit multiple coexisting solutions with different power outputs [26,27]. The appearance of periodic or chaotic solutions, or solutions with single and cross potential well motions, depend on the initial conditions.

The selected four time series (a)-(d) of displacement, velocity and voltage for analysis are presented in Figure 6 to examine a range of vibration patterns.
The system parameters and the output results are shown in Tables 1 and 2, respectively. Interestingly, the power output is relatively small in the case (a) and of comparable level for the other cases (b)-(d). Note that the main difference between (a) and (d) is made by the appearance of nonresonant $(13 \mathrm{~Hz})$ and resonant $(16 \mathrm{~Hz})$ solutions of single well type. On the other hand, the cases (b) and (c) are excited by the larger acceleration amplitude but show more complex response with the appearance of snap-through events. The results of (b)-(d) cases are obtained in the vicinity of the resonance peak 
Table 2. Parameters of chosen cases.

\begin{tabular}{cccccc}
\hline Case & $\begin{array}{c}\text { Vibration } \\
\text { character }\end{array}$ & $\begin{array}{c}\text { Excitation } \\
\text { frequency }\end{array}$ & $\begin{array}{c}\text { Excit. ampl. } \\
\text { (acceleration) }\end{array}$ & $\begin{array}{c}\text { Gap between } \\
\text { repuls. magnets }\end{array}$ & $\begin{array}{c}\text { Load } \\
\text { resist. }\end{array}$ \\
\hline (a) & single well & $13.0 \mathrm{~Hz}$ & $1 \mathrm{~g}$ & $10 \mathrm{~mm}$ & $48 \mathrm{k} \Omega$ \\
(b) & periodic snap-through & $14.4 \mathrm{~Hz}$ & $3 g$ & $10 \mathrm{~mm}$ & $48 \mathrm{k} \Omega$ \\
(c) & chaotic snap-through & $14.0 \mathrm{~Hz}$ & $3 g$ & $10 \mathrm{~mm}$ & $48 \mathrm{k} \Omega$ \\
(d) & single well & $16.0 \mathrm{~Hz}$ & $1 g$ & $15.5 \mathrm{~mm}$ & $48 \mathrm{k} \Omega$ \\
\hline
\end{tabular}

Table 3. Output results in the considered cases (see Fig. 6 for data).

\begin{tabular}{ccc}
\hline Case & $\begin{array}{c}\text { Output } \\
\text { power }[\mathrm{mW}]\end{array}$ & $\begin{array}{c}\text { Displacement } \\
\text { rms amplitude }[\mathrm{mm}]\end{array}$ \\
\hline (a) & 0.001 & 0.96 \\
(b) & 1.548 & 1.95 \\
(c) & 1.797 & 2.21 \\
(d) & 0.598 & 2.09 \\
\hline
\end{tabular}

(Fig. 5). The cases (b) and (c) are similar in response indicating slightly higher level of vibration amplitude and power output for a chaotic response (c).

\section{Solution identification}

\subsection{Wavelets}

Based on the time series $x(t)=\operatorname{disp}(t)$, velocity $(t)$ and voltage $(t)$ for the measured displacement, velocity and voltage, we performed continuous wavelet transformation (CWT) $[28,29]$. The main aim here is to study the system response oscillations in both scales of time and frequency simultaneously. By applying the wavelets to voltage we are able follow the power output in the frequency spectrum.

The corresponding continuous wavelet transform with respect to the wavelet mother function $\psi($.$) is defined as$ follows:

$$
W_{s, n}(x)=\sum_{i=1}^{N} \frac{1}{s} \psi\left(\frac{i-n}{s}\right) \frac{\left(x\left(t_{i}\right)-\langle x\rangle\right)}{\sigma_{x}},
$$

where $\langle x\rangle$ and $\sigma_{x}$ are the averages and standard deviations of corresponding while the letters $s$ and $n$ denote the scale and the time indeces. Finally, the wavelet power spectrum (WPS) of the $x$ time series can be written

$$
P_{W}=\left|W_{s, n}\right|^{2}
$$

In our calculations, we adopted a complex Morlet wavelet as the mother wavelet $[28,29]$. Clearly, the Morlet wavelet consists of a plane wave modulated by a Gaussian function and is described by

$$
\psi(\eta)=\pi^{-1 / 4} e^{\mathrm{i} \theta_{0} \eta} e^{-\eta^{2} / 2},
$$

where $\theta_{0}$, defines the number of oscillations in the wavelet, is the center frequency, frequently referred to as the order of the wavelet, and $\eta$ is a renormalized time variable.

In our analysis, we applied $\theta_{0}=6$ which provides a good balance between the time and frequency resolutions.
In addition, for the above mentioned choice, the scale is approximately equal to the period, and therefore the terms scale and period can be interchanged for interpreting the results [28-30].

The corresponding results of CWT as the wavelet power spectra are presented in Figure 7. A series of plots shows the evolution of power in various periods. Starting from Figure 7a, one can observe well defined maxima (dark red colour) in displacement and velocity channels at the period of 19 sampling units which matches with the excitation frequency $13 \mathrm{~Hz}$. The modulated output voltage (as the detection of the voltage and displacement (velocity) is made at different regions of the beam) is visible in Figure $6 \mathrm{a}$ also contains multiple maxima in voltage plots of Figure $7 \mathrm{a}$. These relatively simple responses are similar to the plots in Figure 7d with a small correction in maximum location which was shifted to the period of 15.6 sampling units which corresponds to $16 \mathrm{~Hz}$. On the other hand in Figures $7 \mathrm{~b}$ and $7 \mathrm{c}$ we observe multiple important periods arranged in periodic (Fig. $7 \mathrm{~b}$ ) and nonperiodic patterns (Fig. 7c). These are related to snap-through responses which are of periodic and chaotic nature, respectively.

\subsection{Phase portraits}

Nonlinear systems are often investigated in terms of the phase portraits and Poincare maps [31]. The corresponding portraits of the examined cases (Fig. 6) are presented in Figure 8. Here we have plotted voltage and velocity against measured displacement. These are two dimensional projections of the larger phase space spanned by all these three coordinates (distance, velocity, voltage) with additional modal components, etc. For better clarity we included stroboscopic points using interval of 12 sampling steps $(0.002 \mathrm{~s})$. This value was used to include all the periods visible in Figures 6 and 7 .

Interestingly, the projections to voltage and velocity against displacement looks similar. From the topological complexity the lines make it possible to distinguish the regularity of solutions. Cases (a) and (d) are relatively simple plots based on a single loop at least in case of velocity-displacement curves. In terms of voltage output, the response shows a simple variation to a single loop in the case (a) while in case (d) is still observable as a single loop. These solutions can be classified as single period harmonic oscillations ((a) and (d) for the velocity-displacement portrait and (d) for the voltage-displacement portrait) or quasi-periodic ((a) for the voltage-displacement portrait). On the other hand case (b) is characterized by a multiple turned loop, but still closed. This indicates the presence of regular, albeit 


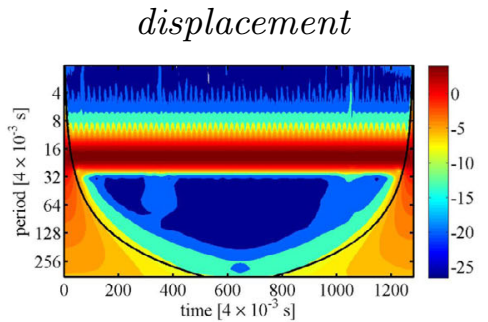

(a) single well

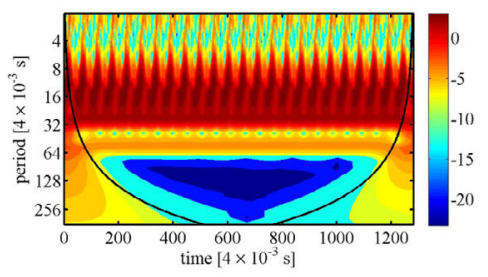

(b) periodic snap-through

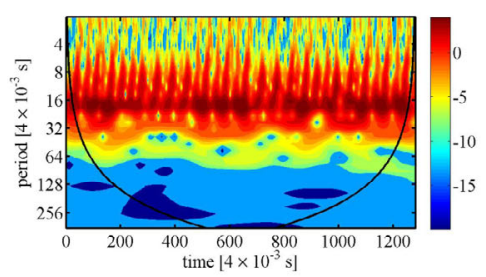

(c) chaotic snap-through

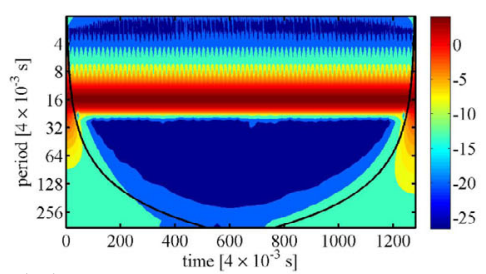

(d) single well
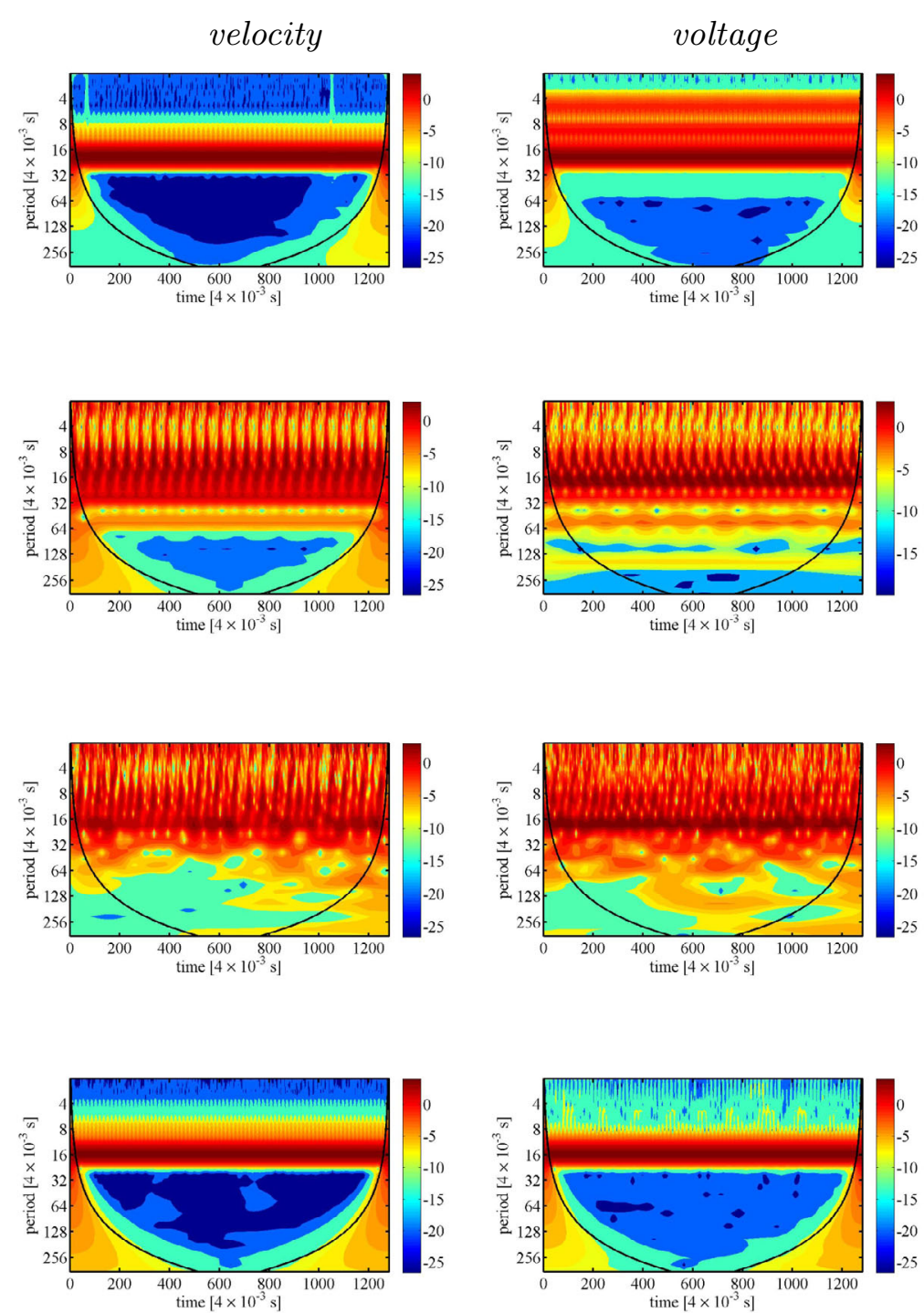

Fig. 7. Wavelet analysis of displacement, velocity and voltage for cases corresponding to solutions presented in Figure 6 . The colors red and blue represent the highest and lowest power levels, respectively, with the other colors denoting intermediate power levels in the logarithmic scale. The black contour lines enclose regions of greater than $95 \%$ confidence for a red noise process, and the region below the thin U-shaped curve denotes the cone of influence (COI). Inside the COI, the edge effects become important, and therefore the results in this region may be unreliable and should be used with caution [29].

multiperiodic, oscillations. Finally, the phase portraits in case (c) show infinitely multiple turn open curve. This implies a chaotic solution. Similarly, the stroboscopic points distribution are characterised by the concentration to singular points or lines, signaling a regular solution or more randomly distributed points characteristic for the chaotic solutions. These stroboscopic points will be also used to estimate the multiscale entropy in the next section.

\subsection{Multiscaled entropy}

Sample entropy analysis is frequently used to improve understanding of the non-linear response of various dynamical systems $[30,32,33]$. This method provides, for measured or simulated signals, a higher level of complexity, defined as "meaningful structural richness" [34,35] in multiple spatio-temporal correlations, for finite length time series.

Goldberger et al. [36] and Costa et al. [37] introduced the concept of multi-scale entropy (MSE) based on a procedure that uses a coarse-grained time series as an average of the original data points within non-overlapping windows by increasing the scale factor $\tau(\tau$ is a natural number) using the following formula (see Fig. 9):

$$
V_{j}^{(\tau)}=\frac{1}{\tau} \sum_{i=n_{j m i n}}^{n_{j m i n}+\tau-1} V_{i}
$$

where upper and lower limit is the above expession are $n_{\text {jmin }}=(j-1) \tau+1$, while $V$ is a raw one-dimensional 

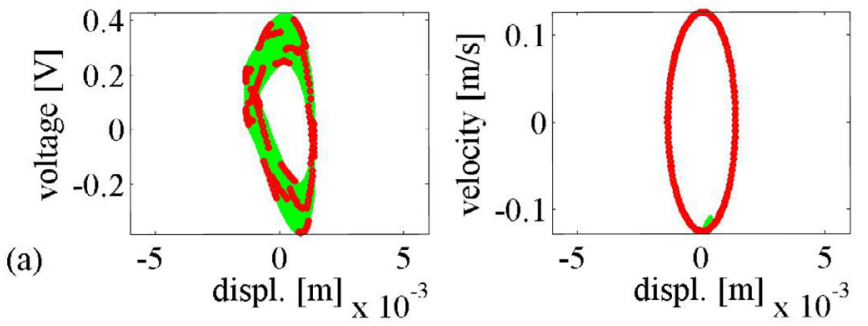

single well

(b)
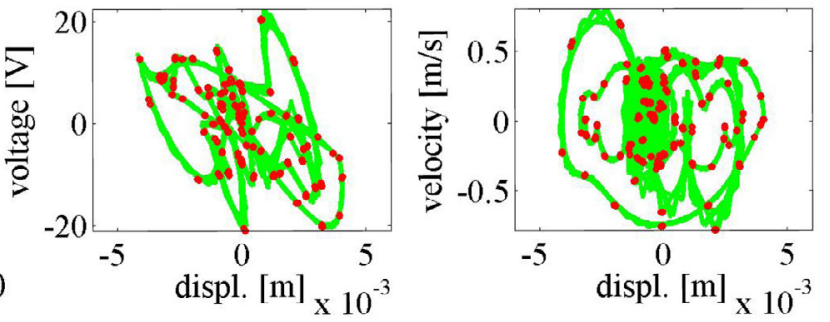

periodic snap-through
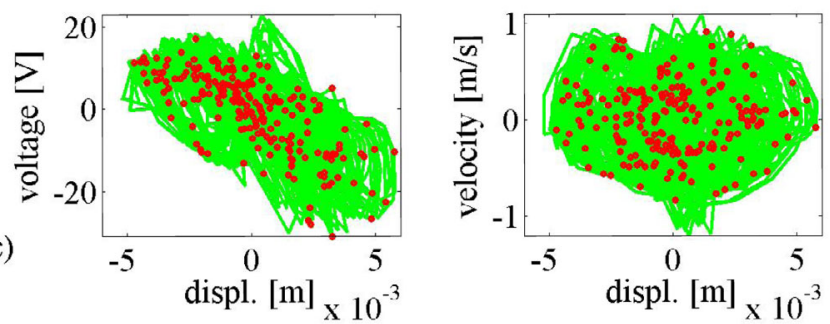

chaotic snap-through
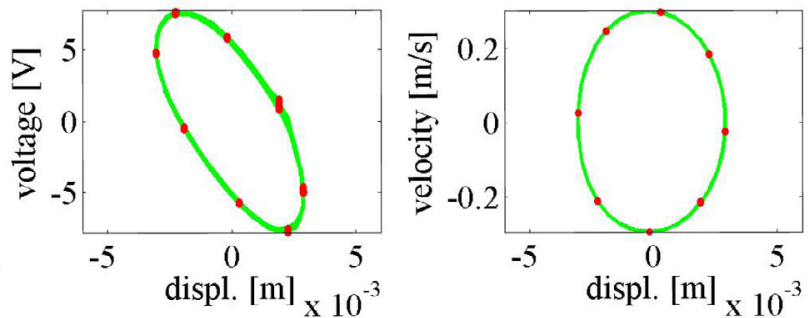

single well

Fig. 8. Phase portraits and the corresponding choice of the consecutive points (cases defined as in Fig. 6).

voltage time series $V=\left\{V_{1}, V_{2}, \ldots, V_{i}, \ldots, V_{N}\right\}$. To avoid oversampling [30], the index $i \in(1, \ldots, N)$ indicates every 12 th element from the original time series (see corresponding points in Fig. 8). In this approach for each scale factor $\tau$, the MSE calculation based on the time series of the coarse-grained $V_{j}^{(\tau)}$ :

$$
\operatorname{MSE}(\mathbf{V}, \tau, m, r)=\operatorname{SampEn}\left(\mathbf{V}^{(\tau)}, m, r\right)
$$

where $m=2$ is the pattern length and $r$ is the similarity factor. For the appropriate dynamics identification, it is usually chosen as $r<\sigma[38,39]$, here $\sigma$ is the standard deviation of the original time series and $V_{i}^{(1)}=V_{i}$.

To estimate $\operatorname{SampEn}\left(\mathbf{V}^{(\tau)}, m, r\right)$ from equation (4) (see also Fig. 9) we count the number of vector pairs denoted by $V_{i}^{(\tau)}$ and $V_{j}^{(\tau)}$ of length $m$ and $m+1$ having distance $d\left[V_{i}^{(\tau)}, V_{j}^{(\tau)}\right]<r$. We denote them by $P_{m}$ and $P_{m+1}$ respectively. According to Richman and Moorman [35]

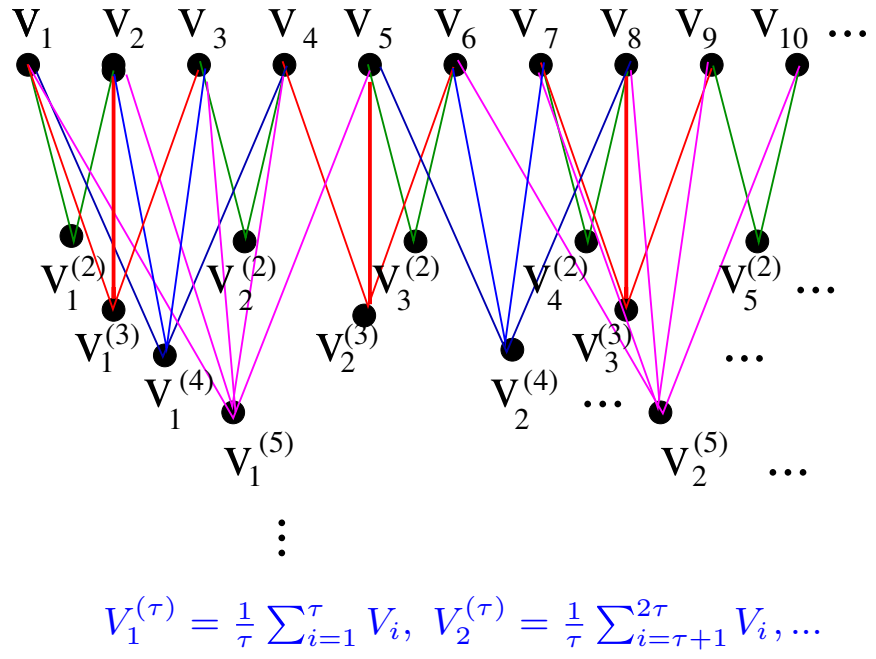

Fig. 9. Scheme of averaging to achieve the effective time series of voltage: $V_{j}^{\tau}$, where $j=1, \ldots$, integer $(N / \tau)$, in the multiscale entropy algorithm (see Eq. (4)). 


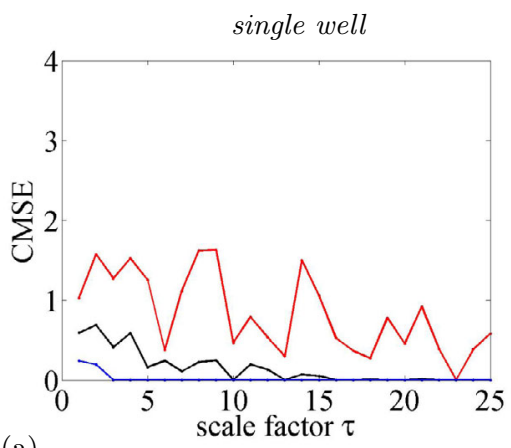

(a)

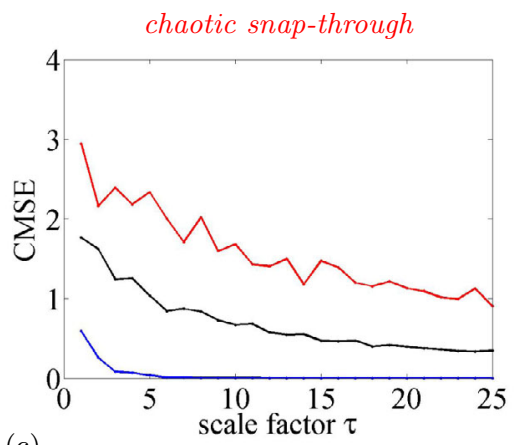

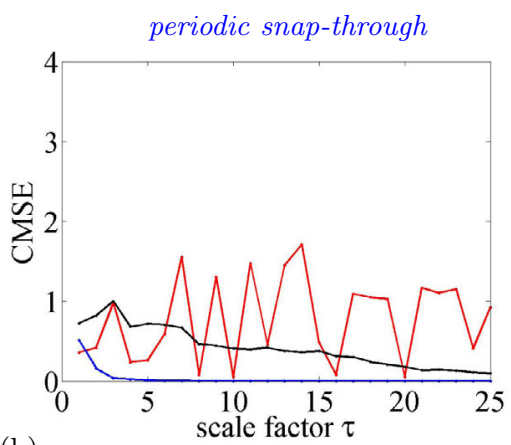

(b)

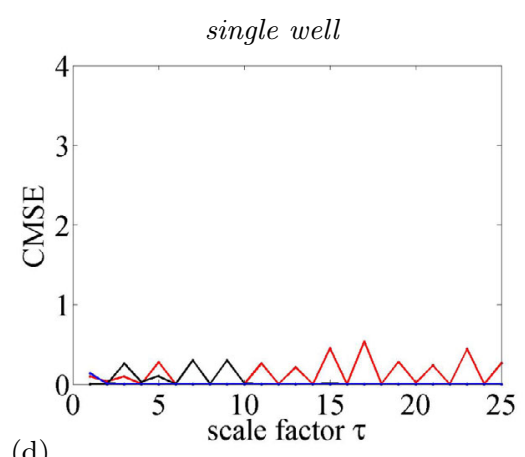

Fig. 10. Multiscale entropy calculated for voltage outputs, $V$, of the considered cases (a)-(d) (cases defined as Fig. 6) for various similarity factors, $r$. Starting from up to down curves (red, black, blue lines): $r=0.04 \sigma_{V}, 0.2 \sigma_{V}$, and $\sigma_{V}$, respectively. $\sigma_{V}$ defines the standard deviation of corresponding voltage time series presented in Figure 6.

the sample entropy is defined:

$$
\operatorname{SampEn}\left(\mathbf{V}^{(\tau)}, m, r\right)=-\log \frac{P_{m+1}}{P_{m}}
$$

which can be considered as the minus of the logarithm of the conditional probability that two sequences with a tolerance $r$ from points (voltages) that remain within $r$ of each other at the next point. Thuraisingham and Gottwald [40] showed that the functional dependency of MSE on the scale factor $\tau$ is highly dependent on the sampling time, and that the same MSE signatures can be found for significantly different dynamic systems.

The method was improved by $\mathrm{Wu}$ et al. [41] who introduced a composite multi-scale entropy (CMSE), which for higher scale factor provides entropy more reliably than the usual multi-scale entropy by including multiple combinations of neighbour points. The formula of the prescribed algorithm for CMSE calculations is described by the following:

$$
\operatorname{CMSE}(\mathbf{V}, \tau, m, r)=\frac{1}{\tau} \sum_{k=1}^{\tau} \operatorname{SampEn}\left(\mathbf{V}^{(\tau, k)}, m, r\right)
$$

where $k$ indicates a different choice of the coarse-grained $\mathbf{V}^{(\tau)}$ from multiple combinations of neighbour elements in the original voltage vector, $\mathbf{V}$.

The results of CMSE analysis estimated for $m=2$ and various similarity factors, $r$, are presented in Figure 10. They should be interpreted in the following way. The results of periodic responses are characterised by small or nodal numbers including also oscillatory dependence against the scale factor $\tau$. The cases (a), (b) and (d) fulfill this criterion. In other case we observe the finite value of entropy which resemble the hyperbola (d). This is an evidence of a chaotic solution.

\section{Conclusions}

The application of a novel broadband bistable laminate beam is considered for piezoelectric-based energy harvesting where the device consists of a bistable cantilever beam with repelling magnets of adjustable distance so that the contribution of the magnets field to the overall strain energy landscape of the device can be controlled. The experimental results such as mechanical resonator velocity, displacement as well as voltage output of the system are studied using wavelet and multiscale entropy in order to better understand the dynamics of this complex system. The examined system response is shown to have different solutions, with the potential to be exploited in a broadband harvesting device. It is noted that the coexisting solutions of a non-linear energy harvester are characterised by different power outputs. To increase the efficiency of the device one has to identify the wave dynamics along the plate and in the region of piezoelectric transducer. We demonstrated that wavelets, phase portraits, and multiscale entropy can provide detailed information of the response characterization. Under open-circuit conditions the voltage and charge is proportional to strain of the piezoelectric macro fibre composite while under 
closed-circuit conditions the current $(d Q / d t)$ is proportional to rate of change of strain. When the load resistance has been optimised to generate maximum power, using $R L=1 / 2 \pi f C$, the system is no longer at these two extremes of boundary conditions and some similarity of voltage across the load resistance with both displacement and velocity can be observed in the wavelet analysis and phase portraits. We have shown that, the signal analysis approach, presented above, can be used to identify dynamic modes and optimise an energy harvesting device in the appropriate switching mechanism between solutions. It is demonstrated that by changing factors such as the magnetic interaction, the characteristics of the bistable laminate harvester, such as the natural frequency, bandwidth, vibration response and peak power can be readily tailored for harvesting

G.L. gratefully acknowledges the support of the Polish National Science Center under Grant No. 2012/05/B/ST8/00080. C.R.B. acknowledges funding from the European Research Council under the European Union's Seventh Framework Programme (FP/2007-2013)/ERC Grant Agreement No. 320963 on Novel Energy Materials, Engineering Science and Integrated Systems (NEMESIS).

\section{References}

1. P.D. Mitcheson, E.M. Yeatman, G.K. Rao, A.S. Holmes, T.C. Green, Proceedings of the IEEE 96, 1457 (2008)

2. R.L. Harne, K.W. Wang, Smart Mat. Struct. 22, 023001 (2013)

3. S.P. Pellegrini, N. Tolou, M. Schenk, J.L. Herder, J. Intell. Mater. Syst. Struct. 24, 1303 (2013)

4. J. Twiefel, H. Westermann, J. Intell. Mater. Syst. Struct. 24, 1291 (2013)

5. M.F. Daqaq, R. Masana, A. Erturk, D.D. Quinn, Appl. Mech. Rev. 66, 040801 (2014)

6. G. Litak, E. Manoach, Eur. Phys. J. Special Topics 222, 1479 (2013)

7. G. Litak, E. Manoach, E. Halvorsen, Eur. Phys. J. Special Topics 224, 2671 (2015)

8. J. Cao, S. Zhou, W. Wang, J. Lin, Appl. Phys. Lett. 106, $173903(2015)$

9. P. Kim, J. Seok, Mechanism and Machine Theory 94, 41 (2015)

10. P. Harris, C.R. Bowen, H.A. Kim, J. Multifunct. Comp. 2, $113(2014)$

11. A. Erturk, D.J. Inman, J. Sound Vibration 330, 2339 (2011)

12. Y. Zhu, J. Zu, J. Intell. Mater. Syst. Struct. 25, 1890 (2014)

13. J. Palosaari, M. Leinonen, J. Juuti, J. Hannu, H. Jantunen, Smart Materials and Structures 23, 085025 (2014)

14. M. Xie, K.C. Aw, W. Gao, Sensors and Actuators A 222, $140(2015)$

15. M. Hyer, J. Compos. Mater. 15, 175 (1981)

16. A.F. Arrieta, P. Hagedorn, A. Ertuk, D.J. Inman, Appl. Phys. Lett. 97, 104102 (2010)

17. D.N. Betts, H.A. Kim, C.R. Bowen, D.J. Inman, Appl. Phys. Lett. 100, 114104 (2012)
18. D.N. Betts, C.R. Bowen, H.A. Kim, N. Gathercole, C.T. Clarke, Eur. Phys. J. Special Topics 222, 1553 (2013)

19. A.F. Arrieta, T. Delpero, A.E. Bergamini, P. Ermanni, Appl. Phys. Lett. 102, 173904 (2013)

20. C.R. Bowen, M. Watson, D.N. Betts, P. Harris, M. Bertin, H.A. Kim, Ferroelectrics 466, 67 (2014)

21. P. Harris, W. Skinner, C.R. Bowen, H.A. Kim, Ferroelectrics 480, 67 (2015)

22. H. Li, F. Dai, S. Du, Smart Materials and Structures 24, 085016 (2015)

23. P. Harris, C.R. Bowen, H.A. Kim, G. Litak, Eur. Phys. J. Plus 131, 109 (2016)

24. P. Harris, G. Litak, J. Iwaniec, C.R. Bowen, Appl. Mech. Mater. 849, 95 (2016)

25. P. Harris, G. Litak, C.R. Bowen, M. Arafa, Proc. SPIE $9865(2016)$

26. A. Syta, C.R. Bowen, H.A. Kim, A. Rysak, G. Litak, Meccanica 50, 1961 (2015)

27. A. Syta, G. Litak, M.I. Friswell, S. Adhikari, Eur. Phys. J. B 89, 99 (2016)

28. P. Kumar, E. Foufoula-Georgiou, Rev. Geophys. 35, 385 (1997)

29. C. Torrence, G.P. Compo, Bull. Amer. Meteor. Soc. 79, 61 (1998)

30. M. Borowiec, A.K. Sen, G. Litak, J. Hunicz, G. Koszalka, A. Niewczas, Forschung im Ingenieurwesen 74, 99 (2010)

31. J.C. Sprott, Chaos and Time-Series Analysis (Oxford University Press, Oxford, 2003)

32. G. Litak, S. Schubert, G. Radons, Nonlinear Dynamics 69, 1255 (2012)

33. M. Borowiec, A. Rysak, D.N. Betts, C.R. Bowen, H.A. Kim, G. Litak, Eur. Phys. J. Plus 129, 211 (2014)

34. P. Grassberger, in Information Dynamics, edited by $\mathrm{H}$. Atmanspacher, H. Scheingraber (Plenum Press, New York, 1991), pp. 15-33

35. J.S. Richman, J.R. Moorman, Am. J. Physiol Heart Circ. Physiol. 278, H2039 (2000)

36. A.L. Goldberger, L.A.N. Amaral, L. Glass, J.M. Hausdorff, P.Ch. Ivanov, R.G. Mark, J.E. Mietus, G.B. Moody, C.-K. Peng, H.E. Stanley, Circulation 101, E215 (2000)

37. M. Costa, A.L. Goldberger, C.-K. Peng, Phys. Rev. Lett. 89, $062102(2002)$

38. M. Costa, C.-K. Peng, A.L. Goldberger, J.M. Hausdorff, Physica A 330, 53 (2003)

39. M. Costa, A.L. Goldberger, C.-K. Peng, Phys. Rev. E 71, $021906(2005)$

40. R.A. Thuraisingham, G.A. Gottwald, Physica A 366, 323 (2006)

41. S.-D. Wu, C.-W. Wu, S.-G. Lin, C.-C. Wang, K.-Y. Lee, Entropy 15, 1069 (2013)

Open Access This is an open access article distributed under the terms of the Creative Commons Attribution License (http://creativecommons.org/licenses/by/4.0), which permits unrestricted use, distribution, and reproduction in any medium, provided the original work is properly cited. 